\title{
Threshold Dynamics of a Huanglongbing Model with Logistic Growth in Periodic Environments
}

\author{
Jianping Wang, Shujing Gao, Yueli Luo, and Dehui Xie \\ Key Laboratory of Jiangxi Province for Numerical Simulation and Emulation Techniques, Gannan Normal University, \\ Ganzhou 341000, China \\ Correspondence should be addressed to Shujing Gao; gaosjmath@tom.com
}

Received 10 January 2014; Accepted 10 February 2014; Published 20 March 2014

Academic Editor: Kaifa Wang

Copyright (C) 2014 Jianping Wang et al. This is an open access article distributed under the Creative Commons Attribution License, which permits unrestricted use, distribution, and reproduction in any medium, provided the original work is properly cited.

We analyze the impact of seasonal activity of psyllid on the dynamics of Huanglongbing (HLB) infection. A new model about HLB transmission with Logistic growth in psyllid insect vectors and periodic coefficients has been investigated. It is shown that the global dynamics are determined by the basic reproduction number $R_{0}$ which is defined through the spectral radius of a linear integral operator. If $R_{0}<1$, then the disease-free periodic solution is globally asymptotically stable and if $R_{0}>1$, then the disease persists. Numerical values of parameters of the model are evaluated taken from the literatures. Furthermore, numerical simulations support our analytical conclusions and the sensitive analysis on the basic reproduction number to the changes of average and amplitude values of the recruitment function of citrus are shown. Finally, some useful comments on controlling the transmission of HLB are given.

\section{Introduction}

Plant disease is an important constraint to crop production. Due to plant diseases, more than $10 \%$ of global food production is lost and 800 million people do not have adequate food in the world [1-3]. Plant pathologists cannot ignore the juxtaposition of these figures for food shortage and the reduction of crops caused by plant disease.

Nowadays, Huanglongbing (HLB) which is a century old disease caused by the bacteria Candidatus Liberibacter spp is one of the most serious problems of citrus worldwide [4]. HLB has been responsible for the near destruction of citrus industries in Asia and Africa [4]. The main symptoms on HLB-infected citrus trees are yellow shoots, leaves with blotchy mottle, and small lopsided fruits $[4,5]$. The HLB is a phloem-restricted, noncultured, Gram-negative bacterium causing crippling diseases denoting "greening" in South Africa, "mottle leaf" in the Philippines, "dieback" in India, and "vein phloem degeneration" in Indonesia. The infected citrus orchards are usually destroyed or become unproductive in 5 to 8 years [4].

Most of the known plant viruses are transmitted by insect vectors and entirely dependent on the behaviour and dispersal capacity of their vectors to spread from plant to plant. HLB, a destructive disease of citrus, can be transmitted by grafting from citrus to citrus and by dodder to periwinkle. The citrus psyllid (Diaphorina Citri Kuwayama) is natural and mainly vector [4]. In this paper, we mainly consider that HLB transmitted from tree to tree by Asian citrus psyllid insect vectors.

Mathematical models play an important role in understanding the epidemiology of vector-transmitted plant diseases. Since the introduction of HLB, a lot of researches have been conducted on the epidemiology of the disease and on the vector, but the result of these two lines of inquiry integrated is very few. Analytical models have also been developed for the spread of citrus canker [6], but models for vectortransmitted bacterial pathogens are still preliminary [7]. In [8], the authors proposed a deterministic compartmental mathematic model to analyze HLB spread between citrus plants. They assumed that all coefficients of the model are constant (autonomous systems). However, in the real world, actual data and evidence show that dynamics of disease transmission are not as simple as shown in the model. In [9], Hall and Hentz have studied seasonal activity of psyllid insect vectors which is correlated with humidity. Seasonal 
fluctuations in the transmission of infectious diseases imply that the corresponding mathematical models may admit periodic solutions. It is interesting and important to study the globally dynamics which are determined by threshold parameter $R_{0}$ in periodic epidemiological models.

Based on above introduction, we propose a model with periodic transmission rates to investigate the seasonal HLB epidemics $[10,11]$. In this model, we consider Logistic growth term for dynamics of susceptible psyllid vector. Furthermore, we assumed that the infective citrus population is generated through susceptible citrus which was bit by infective psyllid and the susceptible psyllid bit the infective citrus which will become infective psyllid. Then, the periodic system is as follows:

$$
\begin{aligned}
\frac{d S_{h}(t)}{d t}= & \Lambda(t)-\beta_{1}(t) S_{h}(t) I_{v}(t)-\mu_{1}(t) S_{h}(t), \\
\frac{d I_{h}(t)}{d t}= & \beta_{1}(t) S_{h}(t) I_{v}(t)-\mu_{1}(t) I_{h}(t)-d(t) I_{h}(t), \\
\frac{d S_{v}(t)}{d t}= & b(t)\left(S_{v}(t)+I_{v}(t)\right)\left[1-\frac{S_{v}(t)+I_{v}(t)}{m\left(S_{h}(t)+I_{h}(t)\right)}\right] \\
& -\beta_{2}(t) S_{v}(t) I_{h}(t), \\
\frac{d I_{v}(t)}{d t}= & \beta_{2}(t) S_{v}(t) I_{h}(t)-\mu_{2}(t) I_{v}(t),
\end{aligned}
$$

with initial condition

$$
S_{h}(0)>0, \quad I_{h}(0)>0, \quad S_{v}(0)>0, \quad I_{v}(0)>0 .
$$

Here, $S_{h}(t), I_{h}(t), S_{v}(t)$, and $I_{v}(t)$ represent susceptible citrus host, infected citrus host, susceptible psyllid, and infected psyllid, respectively. We can easily see that $N_{h}(t)=S_{h}(t)+$ $I_{h}(t)$ and $N_{v}(t)=S_{v}(t)+I_{v}(t)$ are the number of citrus population and psyllid population, respectively. $\Lambda(t)$ is the recruitment rate of citrus at time $t, \beta_{1}(t)$ is the infected rate of citrus host at time $t, \mu_{1}(t)$ and $d(t)$ are the nature death and disease induced death rate of citrus host at time $t$, respectively, $b(t)$ is the intrinsic growth rate of psyllid at time $t, \beta_{2}(t)$ and $\mu_{2}(t)$ are the infected rate and the nature death rate of psyllid at time $t$, respectively, and $m(>0)$ is the maximum abundance of psyllid per citrus. $\Lambda(t), \beta_{1}(t), \mu_{1}(t), d(t), b(t), \beta_{2}(t)$, and $\mu_{2}(t)$ are continuous, positive $\omega$-periodic functions.

The paper is organized as follows. In the next section, we give the basic reproduction number of (1). In Sections 3 and 4 , the results show that the dynamical properties of the model are completely determined by $R_{0}$. That is, if $R_{0}<1$, the disease-free periodic solution is globally asymptotically stable, and if $R_{0}>1$, the model is permanence. In Section 5, we present numerical simulations which demonstrate the theoretical analysis and a brief discussion of our main results.

\section{Basic Reproduction Number}

In the following, we introduce some notations and lemmas which will be used for our further argument.

Let $\left(R^{k}, R_{+}^{k}\right)$ be the standard ordered $k$-dimensional Euclidean space with a norm $\|\cdot\|$. For $u, v \in R^{k}$; we denote $u \geq v$ if $u-v \in R_{+}^{k}, u>v$ if $u-v \in R_{+}^{k} \backslash\{0\}$, and $u \gg v$ if $u-v \in \operatorname{Int}\left(R_{+}^{k}\right)$, respectively.

Define $g^{L}=\max _{t \in[0, \omega)} g(t)$ and $g^{M}=\min _{t \in[0, \omega)} g(t)$, where $g(t)$ is a continuous, positive, $\omega$-periodic function.

Consider the following linear ordinary differential system:

$$
\frac{d x(t)}{d t}=A(t) x(t)
$$

where $A(t)$ is a continuous, cooperative, irreducible, and $\omega$-periodic $k \times k$ matrix function. Denote $\Phi_{A}(t)$ be the fundamental solution matrix of (3) and $r\left(\Phi_{A}(\omega)\right)$ be the spectral radius of $\Phi_{A}(\omega)$. By the Perron-Frobenius Theorem, we know that $r\left(\Phi_{A}(\omega)\right)$ is the principle eigenvalue of $\Phi_{A}(\omega)$; that is, it is simple and admits an eigenvector $v^{*} \gg 0$.

Lemma 1 (see [12]). Let $p=(1 / \omega) \ln r\left(\Phi_{A(\cdot)}(\omega)\right)$. Then there exists a positive $\omega$-periodic function $v(t)$ such that $\exp (p t) v(t)$ is a solution of (3).

Consider the following nonautonomous linear equation:

$$
\frac{d S_{h}(t)}{d t}=\Lambda(t)-\mu_{1}(t) S_{h}(t),
$$

where $\Lambda(t)$ and $\mu_{1}(t)$ are the same as in System (1). From Zhang and Teng ([13, Lemma 2.1]) and simple calculation, we have the following lemma.

Lemma 2. System (4) has a unique positive $\omega$-periodic solution $S_{h}^{*}(t)$ which is globally asymptotically stable.

Consider the following nonautonomous Logistic equation:

$$
\frac{d S_{v}(t)}{d t}=b(t) S_{v}(t)\left(1-\frac{S_{v}(t)}{m S_{h}(t)}\right),
$$

where $b(t)$ and $m$ are the same as in system (1). From Teng and $\mathrm{Li}$ ([14, Lemma 2]) and simple calculation, we can obtain the following lemma.

Lemma 3. System (5) has a unique positive $\omega$-periodic solution $S_{v}^{*}(t)$ which is globally asymptotically stable, where $S_{v}^{*}(t)=$ $m S_{h}^{*}(t)$.

According to Lemmas 2 and 3, it is easy to see that (1) has a unique disease-free periodic solution $\left(S_{h}^{*}(t), 0, S_{v}^{*}(t), 0\right)$.

Now, we use the generation operator approach (see [15]) to derive the basic reproduction number. Applying the symbol of the theory in Wang and Zhao [15], for system (1), we have

$$
\mathscr{F}(t, x)=\left(\begin{array}{c}
\beta_{1}(t) S_{h}(t) I_{v}(t) \\
\beta_{2}(t) S_{v}(t) I_{h}(t) \\
0 \\
0
\end{array}\right) \text {, }
$$




$$
\begin{aligned}
& \mathscr{V}^{+}(t, x)=\left(\begin{array}{c}
0 \\
0 \\
\Lambda(t) \\
b(t)\left(S_{v}(t)+I_{v}(t)\right)
\end{array}\right) \\
& \mathscr{V}^{-}(t, x)=\left(\begin{array}{c}
\left(\mu_{1}(t)+d(t)\right) I_{h}(t) \\
\mu_{2}(t) I_{v}(t) \\
\mu_{1}(t) S_{h}+\beta_{1}(t) S_{h}(t) I_{v}(t) \\
b(t) \frac{\left(S_{v}(t)+I_{v}(t)\right)^{2}}{m\left(S_{h}(t)+I_{h}(t)\right)}+\beta_{2}(t) S_{v}(t) I_{h}(t)
\end{array}\right),
\end{aligned}
$$

where $x=\left(I_{h}(t), I_{v}(t), S_{h}(t), S_{v}(t)\right)^{T}$. Then System (1) can be written as the following form:

$$
\frac{d x(t)}{d t}=\mathscr{F}(t, x(t))-\mathscr{V}(t, x(t)),
$$

where $\mathscr{V}(t, x(t))=\mathscr{V}^{-}(t, x(t))-\mathscr{V}^{+}(t, x(t))$.

It is easy to obtain that the conditions (A1)-(A5) in [15] hold. In the following, we will check the conditions (A6) and (A7) in [15].

We know that $x^{*}(t)=\left(0,0, S_{h}^{*}(t), S_{v}^{*}(t)\right)$ is the diseasefree periodic solution of system (7). Denote

$$
\begin{aligned}
f(t, x(t)) & =\mathscr{F}(t, x(t))-\mathscr{V}(t, x(t)), \\
M(t) & =\left(\frac{\partial f_{i}\left(t, x^{*}(t)\right)}{\partial x_{j}}\right)_{3 \leq i, j \leq 4},
\end{aligned}
$$

where $f_{i}(t, x(t))$ and $x_{i}$ are the $i$ th components of $f(t, x(t))$ and $x$, respectively. According to (6), we have

$$
M(t)=\left(\begin{array}{cc}
-\mu_{1}(t) & 0 \\
\frac{b(t) S_{v}^{* 2}(t)}{m S_{h}^{* 2}(t)} & -b(t)
\end{array}\right) .
$$

It is easy to see that $r\left(\Phi_{M}(\omega)\right)<1$, where $r\left(\Phi_{M}(\omega)\right)$ is the spectral radius of $\Phi_{M}(\omega)$. This implies that $x^{*}(t)$ is linearly asymptotically stable in the disease-free subspace $X_{S}=\left\{\left(0,0, S_{h}, S_{v}\right) \in R_{+}^{4}\right\}$. Thus, condition (A6) in [15] holds.

We further define

$$
\begin{gathered}
F(t)=\left(\frac{\partial \mathscr{F}_{i}\left(t, x^{*}(t)\right)}{\partial x_{j}}\right)_{1 \leq i, j \leq 2}, \\
V(t)=\left(\frac{\partial \mathscr{V}_{i}\left(t, x^{*}(t)\right)}{\partial x_{j}}\right)_{1 \leq i, j \leq 2},
\end{gathered}
$$

where $\mathscr{F}_{i}(t, x)$ and $\mathscr{V}_{i}(t, x)$ are the $i$ th components of $\mathscr{F}(t, x)$ and $\mathscr{V}(t, x)$, respectively. Then, from (6), we obtain that

$$
\begin{aligned}
F(t) & =\left(\begin{array}{cc}
0 & \beta_{1}(t) S_{h}^{*}(t) \\
\beta_{2}(t) S_{v}^{*}(t) & 0
\end{array}\right), \\
V(t) & =\left(\begin{array}{cc}
\mu_{1}(t)+d(t) & 0 \\
0 & \mu_{2}(t)
\end{array}\right) .
\end{aligned}
$$

Let $Y(t, s)$ be a $2 \times 2$ matrix solution of the system:

$$
\begin{gathered}
\frac{d Y(t, s)}{d t}=-V(t) Y(t, s), \quad \forall t \geq s, \\
Y(s, s)=I,
\end{gathered}
$$

where $I$ is $2 \times 2$ identity matrix. From (11) and (12), we have $r\left(\Phi_{-V}(\omega)\right)<1$. Therefore, the condition (A7) in [15] also holds.

Let $C_{\omega}$ be the ordered Banach space of all $\omega$-periodic function from $R \rightarrow R^{2}$, which is equipped with maximum norm $\|\cdot\|_{\infty}$ and the positive cone $C_{\omega}^{+}=\left\{\phi \in C_{\omega}: \phi(t) \geq 0\right.$, for all $t \in R\}$. Define the following linear operator $L: C_{\omega} \rightarrow$ $C_{\omega}$ by

$$
(L \phi)(t)=\int_{0}^{+\infty} Y(t, t-a) F(t-a) \phi(t-a) d a,
$$

$$
\forall t \in R, \quad \phi \in C_{\omega} .
$$

Based on the assumptions above and the results of Wang and Zhang [15], we can derive the basic reproduction number $R_{0}$ of system (1) as follows:

$$
R_{0}=r(L),
$$

and obtain the following conclusion.

Theorem 4. For system (1), the following statements are valid:

(i) $R_{0}=1$ if and only if $r\left(\Phi_{F-V}(\omega)\right)=1$,

(ii) $R_{0}>1$ if and only if $r\left(\Phi_{F-V}(\omega)\right)>1$,

(iii) $R_{0}<1$ if and only if $r\left(\Phi_{F-V}(\omega)\right)<1$,

where $F(t)$ and $V(t)$ are defined in (11).

It follows from Theorem 4 that the disease-free periodic solution $\left(S_{h}^{*}(t), 0, S_{v}^{*}(t), 0\right)$ of system (1) is asymptotically stable if $R_{0}<1$, and it is unstable if $R_{0}>1$.

In order to calculate $R_{0}$, we consider the following linear $\omega$-periodic system:

$$
\frac{d w}{d t}=\left(-V(t)+\frac{1}{\lambda} F(t)\right) w, \quad \lambda \in(0, \infty) .
$$

Let $W(t, s, \lambda), t \geqslant s, s \in R$, be the evolution operator of the System (15) on $R^{2}$. Since $F(t)$ is nonnegative and $-V(t)$ is cooperative, then $r(W(\omega, 0, \lambda))$ is continuous and nonincreasing for $\lambda \in(0, \infty)$, and $\lim _{\lambda \rightarrow \infty} r(W(\omega, 0, \lambda))<1$. Thus, we have the following result, which will be used in our numerical calculation of the basic reproduction ratio $R_{0}$ in Section 5.

Lemma 5 (see [15]). The following statements are valid.

(i) If $r(W(\omega, 0, \lambda))=1$ has a positive solution, $\lambda_{0}$ is an eigenvalue of $L$, and hence $R_{0}>0$.

(ii) If $R_{0}>0$, then $\lambda=R_{0}$ is the unique solution of $r(W(\omega$, $0, \lambda))=1$.

(iii) $R_{0}=0$ if and only if $r(W(\omega, 0, \lambda))<1$ for all $\lambda>0$. 


\section{Global Stability of Disease-Free Periodic Solution}

In this section we will prove the global asymptotical stability of the disease-free periodic solution $\left(S_{h}^{*}(t), 0, S_{v}^{*}(t), 0\right)$.

Let $N_{h}(t)=S_{h}(t)+I_{h}(t), N_{v}(t)=S_{v}(t)+I_{v}(t)$. Denote

$$
\begin{gathered}
\Omega=\left\{\left(S_{h}, I_{h}, S_{v}, I_{v}\right) \in R_{+}^{4} \mid 0 \leq S_{h}+I_{h} \leq N_{1}<+\infty,\right. \\
\left.0 \leq S_{v}+I_{v} \leq N_{2}<+\infty\right\},
\end{gathered}
$$

where $N_{1}=\Lambda^{L} / \mu_{1}^{M}$ and $N_{2}=m N_{1}$. Similar to [16, 17], we firstly prove the following lemmas.

Lemma 6. $\Omega$ is a positively invariant set for (1).

Proof. From the equations in (1), we have

$$
\begin{aligned}
\frac{d N_{h}(t)}{d t} & =\Lambda(t)-\mu_{1}(t) N_{h}(t) \\
& \leq \Lambda^{L}-\mu_{1}^{M} N_{h}(t) \\
& \leq 0 \quad \text { if } N_{h}(t) \geq N_{1}, \\
\frac{d N_{v}(t)}{d t} & =b(t) N_{v}\left(1-\frac{N_{v}}{m N_{h}}\right)-\mu_{2}(t) I_{v}(t) \\
& \leq b(t) N_{v}(t)\left(1-\frac{N_{v}(t)}{m N_{h}}\right) \\
& \leq 0 \quad \text { if } N_{v}(t) \geq N_{2}
\end{aligned}
$$

which implies that $\Omega$ is a positive invariant compact set for (1). The proof is completed.

Lemma 7. Let $\left(S_{h}(t), I_{h}(t), S_{v}(t), I_{v}(t)\right)$ be any solution of system (1). It holds that

$$
\begin{aligned}
& \lim _{t \rightarrow+\infty}\left(N_{h}(t)-S_{h}^{*}(t)\right)=0, \\
& \lim _{t \rightarrow+\infty}\left(N_{v}(t)-S_{v}^{*}(t)\right)=0,
\end{aligned}
$$

where $S_{h}^{*}(t), S_{v}^{*}(t)$ are defined in Lemmas 2 and 3, respectively.

Proof. We denote that $y_{1}(t)=N_{h}(t)-S_{h}^{*}(t)$. It follows from the first equation of (17) that $d y_{1}(t) / d t \leq-\mu_{1}(t) y_{1}(t)$, which implies that $\lim _{t \rightarrow+\infty} y_{1}(t)=\lim _{t \rightarrow+\infty}\left(N_{h}(t)-S_{h}^{*}(t)\right)=0$. Further, from Lemma 6 , we obtain that for any $\varepsilon>0$, there exists a $T>0$ such that

$$
S_{h}^{*}(t)-\varepsilon \leq N_{h}(t) \leq S_{h}^{*}(t)+\varepsilon, \quad N_{v}(t)<N_{2}, \quad \forall t \geq T .
$$

Let $y_{2}(t)=N_{v}(t)-S_{v}^{*}(t)$. From the second equation of (17) and (19), we get

$$
\begin{aligned}
\frac{d y_{2}(t)}{d t}= & b(t) N_{v}(t)\left[1-\frac{N_{v}(t)}{m N_{h}(t)}\right]-\mu_{2}(t) I_{v}(t) \\
& -b(t) S_{v}^{*}(t)\left[1-\frac{S_{v}^{*}(t)}{m S_{h}^{*}(t)}\right]
\end{aligned}
$$

$$
\begin{aligned}
\leq & b(t) N_{v}(t)\left[1-\frac{N_{v}(t)}{m\left(S_{h}^{*}(t)+\varepsilon\right)}\right] \\
& -b(t) S_{v}^{*}(t)\left[1-\frac{S_{v}^{*}(t)}{m S_{h}^{*}(t)}\right] \\
= & b(t)\left(N_{v}(t)-S_{v}^{*}(t)\right)\left[1-\frac{S_{h}^{*}(t)}{S_{h}^{*}(t)+\varepsilon}\right] \\
& -b(t)\left(N_{v}^{*}(t)-S_{v}^{*}(t)\right) \frac{N_{v}(t)}{m\left(S_{h}^{*}(t)+\varepsilon\right)} \\
& +b(t) \frac{S_{v}^{* 2}(t) \varepsilon}{m S_{h}^{*}(t)\left(S_{h}^{*}(t)+\varepsilon\right)} \\
= & -b(t) \frac{N_{v}(t)}{m\left(S_{h}^{*}(t)+\varepsilon\right)} y_{2}(t)+\Delta(\varepsilon)
\end{aligned}
$$

for all $t>T$, where

$$
\begin{aligned}
\Delta(\varepsilon)= & b(t)\left(N_{v}(t)-S_{v}^{*}(t)\right)\left[1-\frac{S_{h}^{*}(t)}{S_{h}^{*}+\varepsilon}\right] \\
& +b(t) \frac{S_{v}^{* 2}(t) \varepsilon}{m S_{h}^{*}(t)\left(S_{h}^{*}+\varepsilon\right)} .
\end{aligned}
$$

Obviously, $\lim _{\varepsilon \rightarrow 0} \Delta(\varepsilon)=0$. Because $\varepsilon$ is arbitrarily small, then $\lim _{t \rightarrow+\infty} y_{2}(t)=\lim _{t \rightarrow+\infty}\left(N_{v}(t)-S_{v}^{*}(t)\right)=0$. Hence, the proof is completed.

Theorem 8. The disease-free periodic solution $\left(S_{h}^{*}(t), 0, S_{v}^{*}(t)\right.$, $0)$ is globally asymptotically stable if $R_{0}<1$, whereas it is unstable if $R_{0}>1$.

Proof. From Theorem 4, we have that $\left(S_{h}^{*}(t), 0, S_{v}^{*}(t), 0\right)$ is unstable if $R_{0}>1$, and $\left(S_{h}^{*}(t), 0, S_{v}^{*}(t), 0\right)$ is locally stable if $R_{0}<1$. Therefore, we only need to show the global attractivity of $\left(S_{h}^{*}(t), 0, S_{v}^{*}(t), 0\right)$ for $R_{0}<1$.

Since $R_{0}<1$, by Theorem 4 , we can choose $\epsilon_{1}>0$ sufficiently small such that

$$
r\left(\Phi_{F-V+M_{\varepsilon_{1}}}(\omega)\right)<1
$$

where

$$
M_{\epsilon_{1}}(t)=\left(\begin{array}{cc}
0 & \epsilon_{1} \\
\epsilon_{1} & 0
\end{array}\right)
$$

From Lemma 6 and (18), we have that, for above mentioned $\epsilon_{1}>0$, there exists a $T_{1}>0$ such that $S_{h}(t) \leq S_{h}^{*}(t)+\epsilon_{1}$, $S_{v}(t) \leq S_{v}^{*}(t)+\epsilon_{1}$ for $t>T_{1}$. It follows from the second and fourth equations that for $t>T_{1}$,

$$
\begin{aligned}
& \frac{d I_{h}(t)}{d t} \leq \beta_{1}(t)\left(S_{h}^{*}(t)+\epsilon_{1}\right) I_{v}(t)-\left(\mu_{1}(t)+d(t)\right) I_{h}(t), \\
& \frac{d I_{v}(t)}{d t} \leq \beta_{2}(t)\left(S_{v}^{*}(t)+\epsilon_{1}\right) I_{h}(t)-\mu_{2}(t) I_{v}(t) .
\end{aligned}
$$


Consider the following comparison system:

$$
\begin{aligned}
& \frac{d \widetilde{I}_{h}(t)}{d t}=\beta_{1}(t)\left(S_{h}^{*}(t)+\epsilon_{1}\right) \tilde{I}_{v}(t)-\left(\mu_{1}(t)+d(t)\right) \tilde{I}_{h}(t), \\
& \frac{d \widetilde{I}_{v}(t)}{d t}=\beta_{2}(t)\left(S_{v}^{*}(t)+\epsilon_{1}\right) \widetilde{I}_{h}(t)-\mu_{2}(t) \widetilde{I}_{v}(t) .
\end{aligned}
$$

In view of Lemma 1, we know that there exists a positive $\omega$ periodic function $v_{1}(t)$ such that $J(t) \leq v_{1}(t) \exp \left(p_{1} t\right)$, where $J(t)=\left(\widetilde{I}_{h}(t), \widetilde{I}_{v}(t)\right)^{T}$ and $p_{1}=(1 / \omega) \ln r\left(\Phi_{F-V+M_{\epsilon}}(\omega)\right)<$ 0 . It follows from (22) that $\lim _{t \rightarrow+\infty} \widetilde{I}_{h}(t)=0$ and $\lim _{t \rightarrow+\infty} \widetilde{I}_{v}(t)=0$. By the comparison of theorem [18], we have $\lim _{t \rightarrow+\infty} I_{h}(t)=0$ and $\lim _{t \rightarrow+\infty} I_{v}(t)=0$. From (18), we have

$$
\begin{aligned}
& \lim _{t \rightarrow+\infty}\left(S_{h}(t)-S_{h}^{*}(t)\right)=0, \\
& \lim _{t \rightarrow+\infty}\left(S_{v}(t)-S_{v}^{*}(t)\right)=0 .
\end{aligned}
$$

Hence, the disease free periodic solution $\left(S_{h}^{*}(t), 0, S_{v}^{*}(t), 0\right)$ is globally attractive. This completes the proof.

\section{Permanence}

In this section, we show that if $R_{0}>1$, then the disease persists.

Firstly, we define $X=\left\{\left(S_{h}, I_{h}, S_{v}, I_{v}\right) \in R_{+}^{4}\right\}, X_{0}=\left\{\left(S_{h}, I_{h}\right.\right.$, $\left.\left.S_{v}, I_{v}\right) \in X: S_{h} \geq 0, I_{h}>0, S_{v} \geq 0, I_{v}>0\right\}$, and $\partial X_{0}=$ $X \backslash X_{0}$, and we denote $u\left(t, x_{0}\right)$ as the unique solution of System

(1) with the initial value $x_{0}=\left(S_{h}^{0}, I_{h}^{0}, S_{v}^{0}, I_{v}^{0}\right)$.

Define Poincaré map $P: X \rightarrow X$ associated with System (1) as follows:

$$
P\left(x_{0}\right)=u\left(\omega, x_{0}\right), \quad \forall x_{0} \in X
$$

By Lemma 6, it is easy to see that both $X$ and $X_{0}$ are positively invariant and $P$ is point dissipative. Set

$$
\begin{gathered}
M_{\partial}=\left\{\left(S_{h}^{0}, I_{h}^{0}, S_{v}^{0}, I_{v}^{0}\right) \in \partial X_{0} \mid P^{m}\left(S_{h}^{0}, I_{h}^{0}, S_{v}^{0}, I_{v}^{0}\right) \in \partial X_{0},\right. \\
\left.m \in Z^{+}\right\}
\end{gathered}
$$

where $Z^{+}=\{0,1,2, \ldots\}$. We claim that

$$
M_{\partial}=\left\{\left(S_{h}, 0, S_{v}, 0\right), S_{h} \geq 0, S_{v} \geq 0\right\} .
$$

Obviously, $M_{\partial} \supseteq\left\{\left(S_{h}, 0, S_{v}, 0\right), S_{h} \geq 0, S_{v} \geq 0\right\}$. Next we want to show $M_{\partial} \backslash\left\{\left(S_{h}, 0, S_{v}, 0\right), S_{h} \geq 0, S_{v} \geq 0\right\}=\emptyset$. If it does not hold, then there exists a point $\left(S_{h}^{0}, I_{h}^{0}, S_{v}^{0}, I_{v}^{0}\right) \in M_{\partial} \backslash$ $\left\{\left(S_{h}, 0, S_{v}, 0\right), S_{h} \geq 0, S_{v} \geq 0\right\}$.

Case 1. $I_{h}^{0}=0$ and $I_{v}^{0}>0$. It is obvious that $I_{v}(t)>0$ and $S_{h}(t)>0$ for any $t>0$. Then, from the second equation of System (1), $d I_{h}(t) /\left.d t\right|_{t=0}=\beta_{1}(0) S_{h}(0) I_{v}(0)>0$ holds. It follows that $\left(S_{h}(t), I_{h}(t), S_{v}(t), I_{v}(t)\right) \notin \partial X_{0}$ for $0<t \ll 1$. This is a contradiction.
Case 2. $I_{h}^{0}>0$ and $I_{v}^{0}=0$. It is obvious that $I_{h}(t)>0$ and $S_{v}(t)>0$ for any $t>0$. Then, from the fourth equation of System (1), $d I_{v}(t) /\left.d t\right|_{t=0}=\beta_{2}(0) S_{v}(0) I_{h}(0)>0$ holds. It follows that $\left(S_{h}(t), I_{h}(t), S_{v}(t), I_{v}(t)\right) \notin \partial X_{0}$ for $0<t \ll 1$. This is a contradiction.

That is to say, for any $\left(S_{h}^{0}, I_{h}^{0}, S_{v}^{0}, I_{v}^{0}\right) \notin\left\{\left(S_{h}, 0, S_{v}, 0\right): S_{h} \geq\right.$ $\left.0, S_{v} \geq 0\right\}$, then $\left(S_{h}^{0}, I_{h}^{0}, S_{v}^{0}, I_{v}^{0}\right) \notin M_{\partial}$. Therefore we have $M_{\partial}=$ $\left\{\left(S_{h}, 0, S_{v}, 0\right): S_{h} \geq 0, S_{v} \geq 0\right\}$.

Next, we present the following result of the uniform persistence of the disease.

Theorem 9. Suppose $R_{0}>1$. Then there is a positive constant $\epsilon>0$ such that each positive solution $\left(S_{h}(t), I_{h}(t), S_{v}(t), I_{v}(t)\right)$ of System (1) satisfies

$$
\liminf _{t \rightarrow+\infty} I_{h}(t) \geq \epsilon, \quad \liminf _{t \rightarrow+\infty} I_{v}(t) \geq \epsilon .
$$

Proof. By Theorem 4, we obtain $r\left(\Phi_{F-V}(\omega)\right)>1$. So we can choose $\eta>0$ small enough such that $r\left(\Phi_{F-V-M_{\eta}}\right)>1$, where

$$
M_{\eta}=\left(\begin{array}{ll}
0 & \eta \\
\eta & 0
\end{array}\right) .
$$

Put $P_{0}=\left\{S_{h}^{*}(0), 0, S_{v}^{*}(0), 0\right\}$. Now we proceed by contradiction to prove that

$$
\limsup _{m \rightarrow+\infty} d\left(P^{m}\left(S_{h}^{0}, I_{h}^{0}, S_{v}^{0}, I_{v}^{0}\right), P_{0}\right) \geq \delta .
$$

If it does not hold, then

$$
\limsup _{m \rightarrow+\infty} d\left(P^{m}\left(S_{h}^{0}, I_{h}^{0}, S_{v}^{0}, I_{v}^{0}\right), P_{0}\right)<\delta
$$

for some $\left(S_{h}^{0}, I_{h}^{0}, S_{v}^{0}, I_{v}^{0}\right) \in X_{0}$. Without loss of generality, suppose that

$$
d\left(P^{m}\left(S_{h}^{0}, I_{h}^{0}, S_{v}^{0}, I_{v}^{0}\right), P_{0}\right)<\delta, \quad \forall m \in Z_{+} .
$$

By the continuity of the solutions with respect to the initial values, we obtain

$$
\begin{array}{r}
\left\|u\left(t, P^{m}\left(S_{h}^{0}, I_{h}^{0}, S_{v}^{0}, I_{v}^{0}\right)\right)-u\left(t, P_{0}\right)\right\| \leq \eta \\
\forall t \in[0, \omega], \quad \forall m \in Z_{+} .
\end{array}
$$

For any $t \geq 0$, there exists a $m \in Z_{+}$such that $t=m \omega+t_{1}$, where $t_{1} \in[0, \omega]$. Then we have

$$
\begin{aligned}
& \left\|u\left(t,\left(S_{h}^{0}, I_{h}^{0}, S_{v}^{0}, I_{v}^{0}\right)\right)-u\left(t, P_{0}\right)\right\| \\
& \quad=\left\|u\left(t_{1}, P^{m}\left(S_{h}^{0}, I_{h}^{0}, S_{v}^{0}, I_{v}^{0}\right)\right)-u\left(t_{1}, P_{0}\right)\right\| \leq \eta
\end{aligned}
$$

for all $t \geq 0$, which implies that $S_{h}^{*}(t)-\eta \leq S_{h}(t) \leq S_{h}^{*}(t)+\eta$, $S_{v}^{*}(t)-\eta \leq S_{v}(t) \leq S_{v}^{*}(t)+\eta$. Then from (1) we have

$$
\begin{aligned}
& \frac{d I_{h}(t)}{d t} \geq \beta_{1}(t)\left(S_{h}^{*}(t)-\eta\right) I_{v}(t)-\left(\mu_{1}(t)+d(t)\right) I_{h}(t), \\
& \frac{d I_{v}(t)}{d t} \geq \beta_{2}(t)\left(S_{v}^{*}(t)-\eta\right) I_{h}(t)-\mu_{2}(t) I_{v}(t) .
\end{aligned}
$$


TABle 1: Parameter definitions and values used for numerical simulations of the Huanglongbing model.

\begin{tabular}{|c|c|c|c|c|}
\hline Parameter & Definition & Average value & Unit & Reference \\
\hline$\Lambda$ & The recruitment rate of citrus & - & month $^{-1}$ & Estimate \\
\hline$\beta_{1}$ & Infected rate of citrus & - & month $^{-1}$ & Estimate \\
\hline$\mu_{1}$ & Nature death rate of citrus & $0.00275-0.004167$ & month $^{-1}$ & [20] \\
\hline$d$ & Disease induced death rate of citrus & $0.016667-0.027775$ & month $^{-1}$ & {$[21]$} \\
\hline$D$ & Birth rate of psyllid & $3.78327-33.526137$ & month $^{-1}$ & {$[20,22]$} \\
\hline$\beta_{2}$ & Infected rate of psyllid & - & month $^{-1}$ & Estimate \\
\hline$\mu_{2}$ & Nature death rate of psyllid & $0.1169825-0.95052$ & month $^{-1}$ & [23] \\
\hline$m$ & Max abundance of psyllid per citrus & $120-1000$ & - & {$[24]$} \\
\hline
\end{tabular}

TABLE 2: Parameter functions for model (1) according to the values of Table 1.

\begin{tabular}{lcr}
\hline Parameter functions & Value & Reference \\
\hline$\beta_{1}(t)$ & $0.0042925+0.003543 \cos (2 \pi t / 12)$ & Estimate \\
$\mu_{1}(t)$ & $0.0034585+0.0007085 \cos (2 \pi t / 12)$ & {$[20]$} \\
$d(t)$ & $0.022221+0.005554 \cos (2 \pi t / 12)$ & {$[21]$} \\
$D(t)$ & $18.6547035+14.8714335 \cos (2 \pi t / 12)$ & Estimate \\
$\beta_{2}(t)$ & $0.008779171+0.004838437 \cos (2 \pi t / 12)$ & {$[23]$} \\
$\mu_{2}(t)$ & $0.53375125+0.41676875 \cos (2 \pi t / 12)$ & {$[20,22,23]$} \\
$b(t)=D(t)-\mu_{2}(t)$ & $18.120952+14.45466475 \cos (2 \pi t / 12)$ & {$[24]$} \\
$m$
\end{tabular}

Consider the linear system

$$
\begin{aligned}
& \frac{d \widehat{I}_{h}(t)}{d t}=\beta_{1}(t)\left(S_{h}^{*}(t)-\eta\right) \widehat{I}_{v}(t)-\left(\mu_{1}(t)+d(t)\right) \widehat{I}_{h}(t) \\
& \frac{d \widehat{I}_{v}(t)}{d t}=\beta_{2}(t)\left(S_{v}^{*}(t)-\eta\right) \widehat{I}_{h}(t)-\mu_{2}(t) \widehat{I}_{v}(t) .
\end{aligned}
$$

By Lemma 1 and the standard comparison principle, we have that there exists a positive $\omega$-periodic function $v_{2}(t)$ such that $J(t)=\exp \left(p_{2} t\right) v_{2}(t)$ is a solution of System (38), where $J(t)=$ $\left(\widehat{I}_{h}(t), \widehat{I}_{v}(t)\right)^{T}$ and

$$
p_{2}=\frac{1}{\omega} \ln r\left(\Phi_{F-V-M_{\eta}}(\omega)\right) .
$$

It follows from $r\left(\Phi_{F-V-M_{\eta}}(\omega)\right)>1$ that $p_{2}>0$ and $J(t) \rightarrow$ $+\infty$ as $t \rightarrow+\infty$. Applying the comparison principle [18], we know that $I_{h}(t) \rightarrow+\infty$ and $I_{v}(t) \rightarrow+\infty$ as $t \rightarrow+\infty$. This is a contradiction. Thus, we have proved that (32) holds and $P$ is weakly uniformly persistent with respect to $\left(X_{0}, \partial X_{0}\right)$.

According to the results of Lemma 7, we can easily obtain that $P$ has a global attractor $P_{0}$. It is easy to obtain that $P_{0}$ is an isolated invariant set in $X$ and $W^{s}\left(P_{0}\right) \cap X_{0}=\emptyset$. We know that $P_{0}$ is acyclic in $M_{\partial}$ and every solution in $M_{\partial}$ converges to $P_{0}$. According to Zhao [19], we have that $P$ is uniformly persistent with respect to $\left(X_{0}, \partial X_{0}\right)$. This implies that the solution of (1) is uniformly persistent with respect to $\left(X_{0}, \partial X_{0}\right)$. Thus we have that there exists a $\epsilon>0$ such that $\liminf _{t \rightarrow+\infty} I_{h}(t) \geq \epsilon$, $\lim \inf _{t \rightarrow+\infty} I_{v}(t) \geq \epsilon$.

\section{Numerical Simulations and Sensitivity Analysis}

In this section, we will make numerical simulations by means of Matlab 7.1 to support our theoretical results, to predict the trend of the disease, and to explore some control and prevention measures. Numerical values of parameters of system (1) are given in Table 1 (most of the data are taken from the literatures $([20-24]))$.

According to the periodicity of System (1) and Table 1, we set $\mu_{1}(t)=\alpha_{1}^{0}+\alpha_{2}^{0} \cos (2 \pi t / 12)$, where $\alpha_{2}^{0}=(0.004167-$ $0.00275) / 2=0.0007085$ and $\alpha_{1}^{0}=0.00275+\alpha_{2}^{0}=0.0034585$. By the similar method, we can obtain the other parameter functions of model (1) (see Table 2). For the simulations that follows, we apply the parameters in Table 2 unless otherwise stated.

Choose $\Lambda(t)=0.00265+0.00235 \cos (2 \pi t / 12)$. Then from Lemma 5 , we can compute $R_{0}=0.9844<1$ by means of Matlab 7.1. From Theorem 8 we obtain that the infected citrus population $I_{h}(t)$ and the infected psyllid population $I_{v}(t)$ of system (1) are extinct (see Figures 1 and 2).

Choose $\Lambda(t)=0.005+0.0035 \cos (2 \pi t / 12)$. Then from Lemma 5, we obtain that $R_{0}=1.8342>1$. From Theorem 9 we have that the infected citrus population $I_{h}(t)$ and the infected psyllid population $I_{v}(t)$ of System (1) are permanence (see Figures 3 and 4).

From the formulae for $R_{0}$, we can predict the general tendency of the epidemic in a long term according to the current situation, which is presented in Figures 1, 2, 3, and 4. From the first two figures we know that the epidemic of 


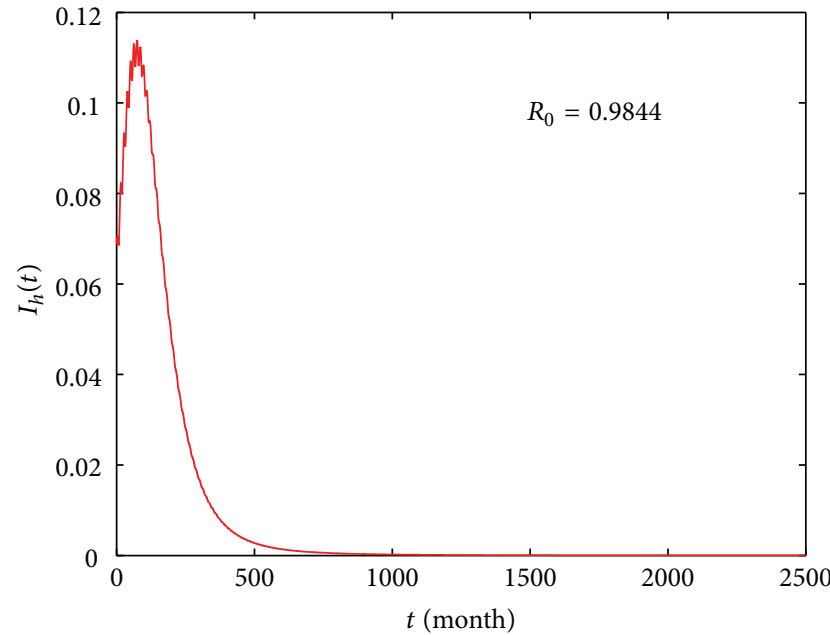

(a)

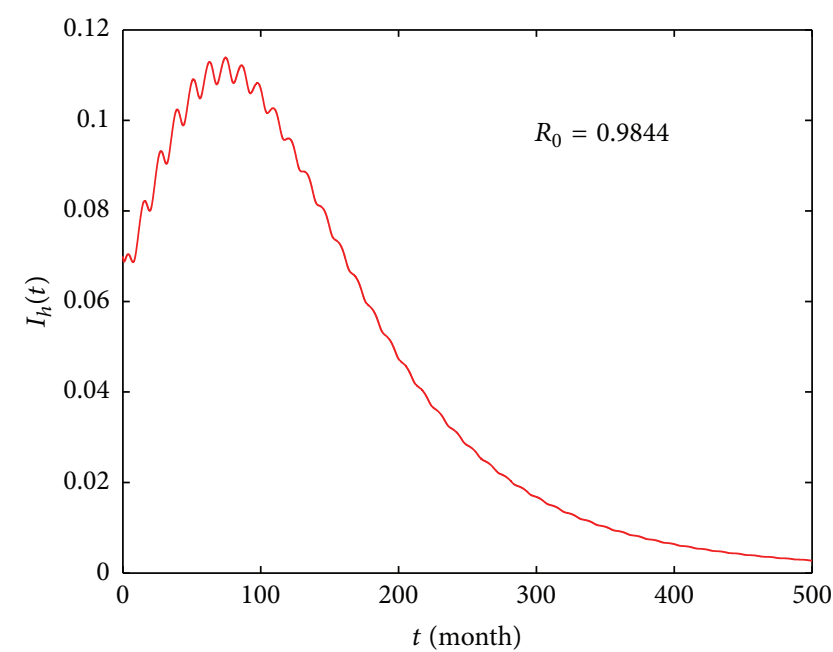

(b)

FIGURE 1: Graphs of numerical simulations of (1) showings the tendency of the infected citrus population. (a) $t \in[0,2500]$; (b) $t \in[0,500]$.

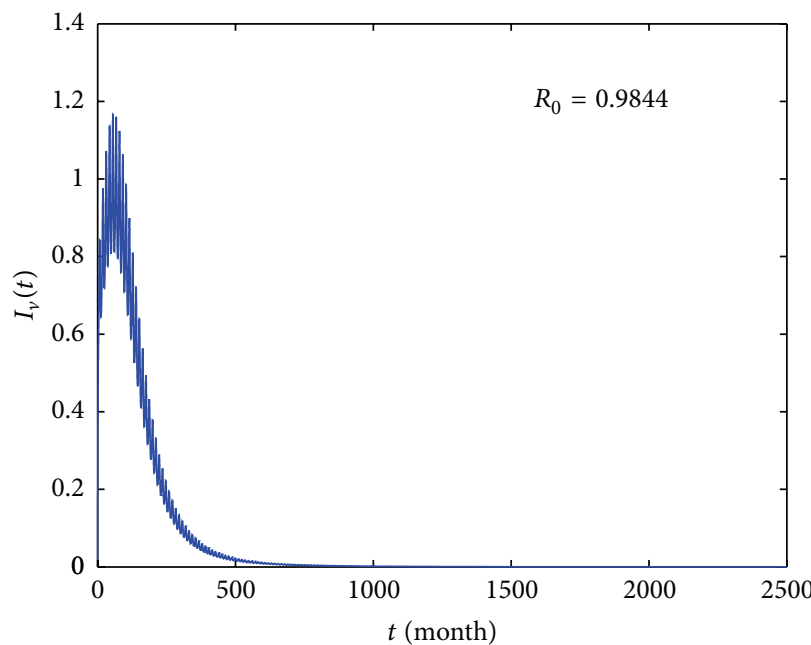

(a)

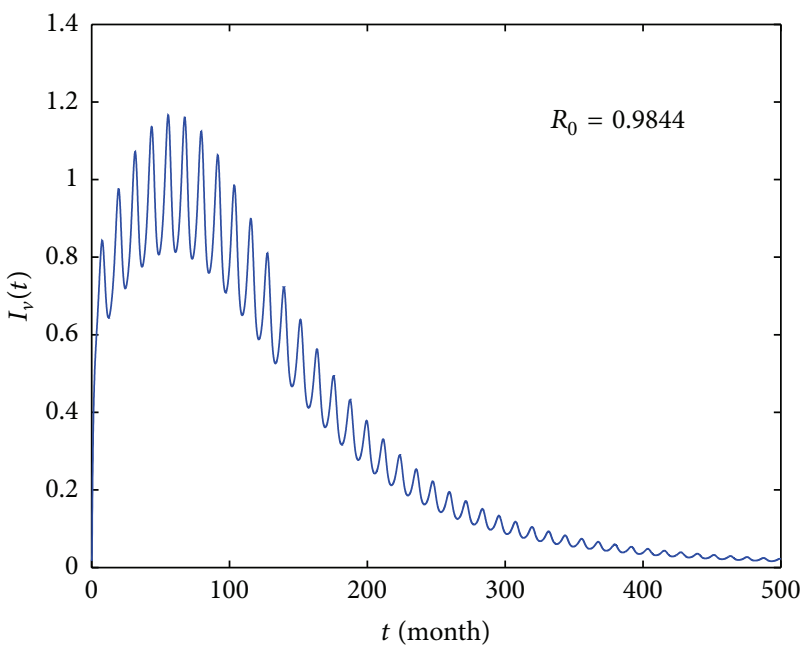

(b)

FIGURE 2: It is similar to Figure 1.

Huanglongbing can be rising in a short time but cannot be outbreak with the current prevention and control measures. From Figures 3 and 4, we can see that the epidemic of Huanglongbing dropped heavily after 100 months, while there is still tendency to a stable periodic solution in a long time.

Next, we perform some sensitivity analysis to determine the influence $R_{0}$ on the parameters $\Lambda(t), \beta_{1}(t)$, and $\beta_{2}(t)$.

We choose function $\Lambda(t)=\Lambda_{1}^{0}+\Lambda_{2}^{0} \cos (2 \pi t / 12)$, where $\Lambda_{1}^{0}, \Lambda_{2}^{0}$ denote the average and amplitude values of $\Lambda(t)$, respectively, and $\Lambda_{1}^{0}=(1 / 12) \int_{0}^{12} \Lambda(t) d t$. From Figure 5, we can observe that the blue line is linear relation between $R_{0}$ and $\Lambda_{2}^{0}$, and $R_{0}$ increases as $\Lambda_{2}^{0}$ increases. The red curve reflects the influence of the average value of $\Lambda(t)$ on $R_{0}$. Figure 5 shows that $\Lambda_{1}^{0}$ is more sensitive than $\Lambda_{2}^{0}$ on the basic reproduction number $R_{0}$. Therefore, in the real world, decreasing the average recruitment rate of citrus is the valuable way to control Huanglongbing.

Now, we consider the combined influence of $\beta_{1}(t)$ and $\beta_{2}(t)$ on $R_{0}$. Set $\Lambda(t)=0.0027+0.00235 \cos (2 \pi t / 12)$, $\beta_{1}(t)=a_{1}+b_{1} \cos (2 \pi t / 12)$ and $\beta_{2}(t)=a_{2}+b_{2} \cos (2 \pi t / 12)$. Moreover, we know that $a_{1}=(1 / 12) \int_{0}^{12} \beta_{1}(t) d t$ and $a_{2}=(1 / 12) \int_{0}^{12} \beta_{2}(t) d t$. Other parameters can be seen in Table 2.

Case (I). We fix $b_{1}=0.003543$ and $b_{2}=0.004838437$, and let $a_{1}$ vary from 0.00001 to 0.015 and $a_{2}$ from 0.00001 to 0.02 . For this case, it is interesting to examine how the average values of adequate contact rate $\beta_{1}(t)$ and $\beta_{2}(t)$ affect the basic reproduction number $R_{0}$. Numerical results shown 


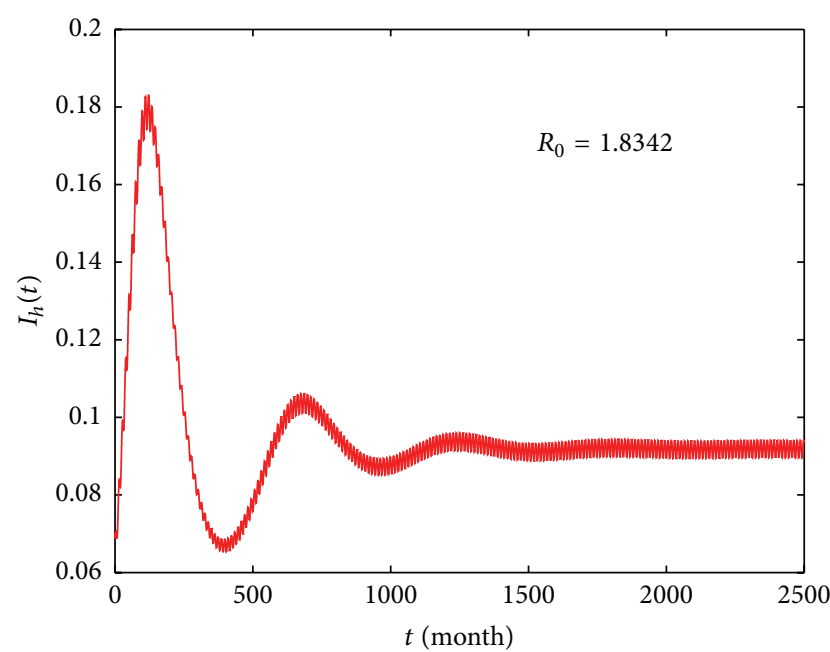

(a)

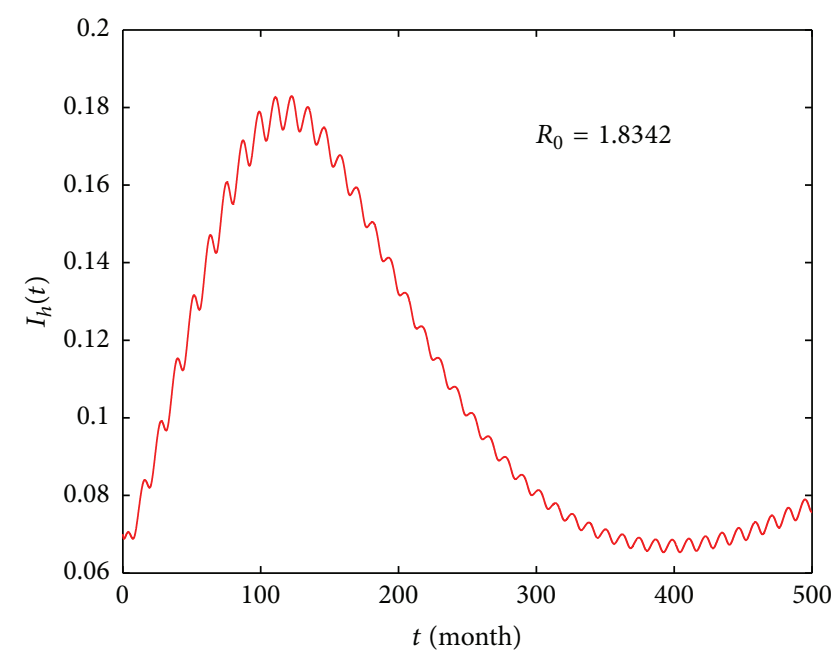

(b)

FIGURE 3: The figures show that the infected citrus population is permanence. (a) $t \in[0,2500]$; (b) $t \in[0,500]$.

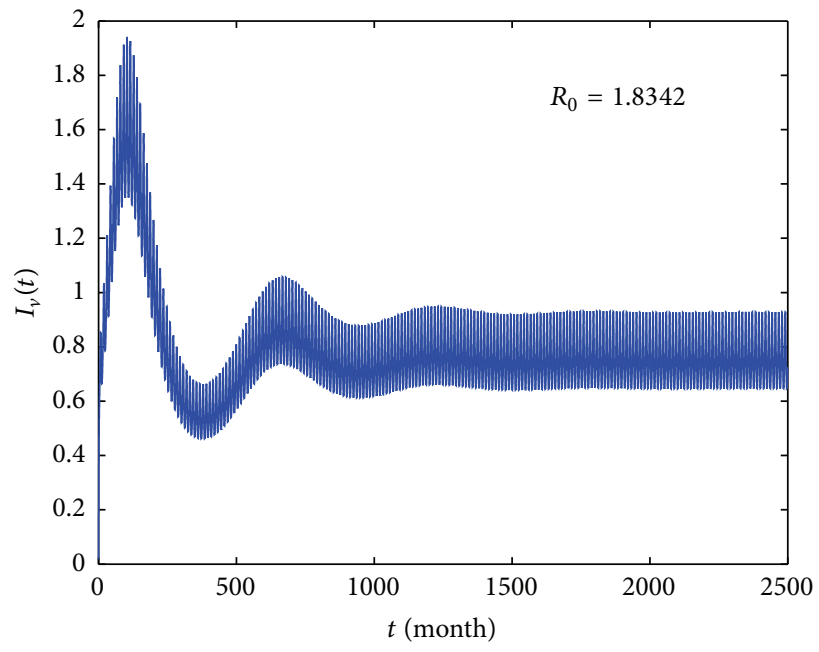

(a)

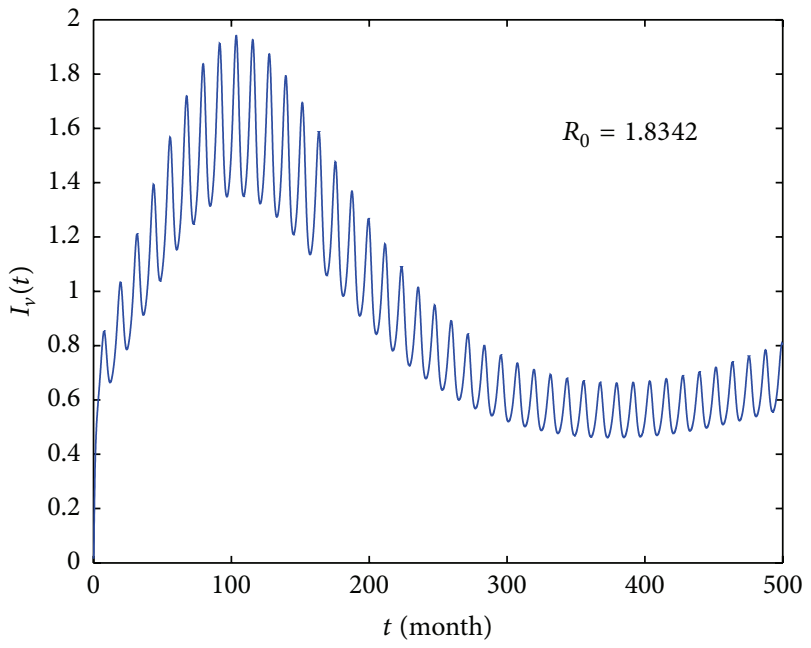

(b)

Figure 4: It is similar to Figure 3.

in Figure 6 imply that the basic reproduction number $R_{0}$ may be less than 1 when $a_{1}$ or $a_{2}$ is small enough. And the results also imply that $R_{0}$ increases as $a_{1}$ and $a_{2}$ increase. Further, we can observe that from Figure 6(i)the smaller the values of $a_{1}$ or $a_{2}$ are, the more sensitive $R_{0}$ is; (ii) increasing $a_{2}$ may be more sensitive for $R_{0}$ when $a_{1}$ is fixed; (iii) increasing $a_{1}$ may be more sensitive for $R_{0}$ when $a_{2}$ is fixed.

Case (II). We fix $a_{1}=0.0042925$ and $a_{2}=0.00877917$, and let $b_{1}$ vary from 0.000001 to 0.005 and $b_{2}$ from 0.000002 to 0.006 . Then we obtain the result of numerical simulation and it is shown in Figure 7. Obviously, Figure 7 shows that $R_{0}$ is linearly related to both $b_{1}$ and $b_{2}$ with the pattern that $R_{0}$ decreases to a relatively small value (less than 1 ) only when $b_{1}$ and $b_{2}$ are very small.
By the above graphs of the basic reproduction number $R_{0}$ on the average values of recruitment rate of citrus $\Lambda(t)$ and adequate contact rate $\beta_{1}(t), \beta_{2}(t)$, we know that the basic reproduction number $R_{0}$ is a monotonic increasing function by the average values. From the sensitivity analysis diagrams, we observe that $R_{0}$ falls to less than 1 by decreasing the values of those parameters.

\section{Conclusion}

In this paper, we have analyzed a HLB transmission model with Logistic growth in periodic environments. It is proved that $R_{0}$ is the threshold for distinguishing the disease extinction or permanence. The disease-free periodic solution is 


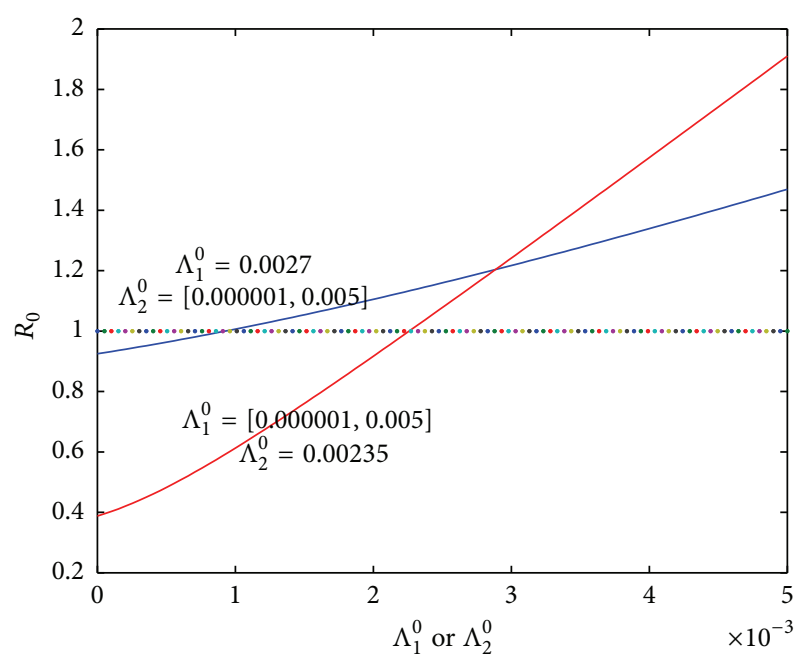

FIGURE 5: The graph shows the sensitivity of the basic reproduction number $R_{0}$ to the changes of $\Lambda(t)$.

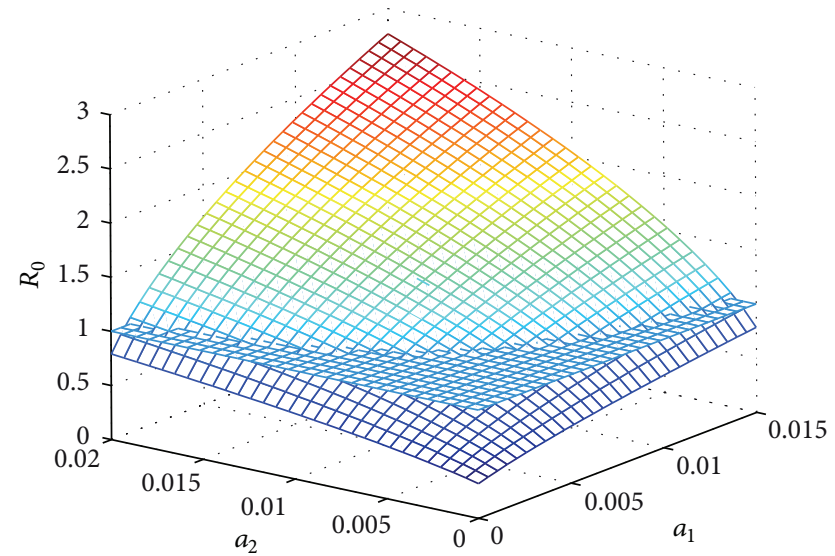

FIGURE 6: The graph of $R_{0}$ in terms of $a_{1}$ and $a_{2}$.

globally asymptotically stable and the disease dies out when $R_{0}<1$. When $R_{0}>1$, the disease persists.

The numerical simulations shown in Figure 5 show that there are some parameter ranges of $\Lambda_{1}$ and $\Lambda_{2}$ such that the threshold parameter $R_{0}$ is smaller than 1 . It indicates a useful way to eradicate Huanglongbing by limiting the recruitment of citrus, including the average value and amplitude of recruitment function.

The results shown in Figure 6 (Figure 7) show that if the amplitudes of infected functions $b_{1}, b_{2}$ (the average infected rate $a_{1}, a_{2}$ ) are fixed, we can control the infection of citrus and psyllid by limiting the average infected rates $a_{1}, a_{2}$ (the amplitudes of infected functions $b_{1}, b_{2}$ ).

According to the above theoretical analysis and numerical simulations, we can conclude that the recruitment of citrus and the infection of citrus and psyllid have significant effects on Huanglongbing transmission. In order to prevent the epidemic disease from generating endemic, making an appropriate reduction of the recruitment rate of citrus and

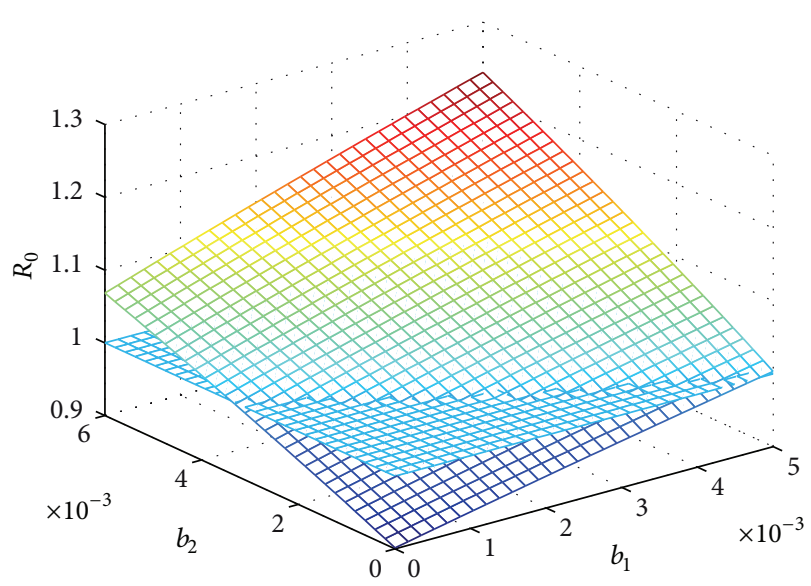

FIGURE 7: The graph of $R_{0}$ in terms of $b_{1}$ and $b_{2}$.

decreasing the contact rate between psyllid and the citrus are effective measures to control Huanglongbing.

\section{Disclosure}

The paper is approved by all authors for publication. The authors would like to declare that the work described was original research that has not been published previously and not under consideration for publication elsewhere.

\section{Conflict of Interests}

No conflict of interests exists in the submission of this paper.

\section{Acknowledgments}

The research has been supported by the Natural Science Foundation of China (11261004), the Natural Science Foundation of Jiangxi Province (20122BAB211010), the Science and Technology Plan Projects of Jiangxi Provincial Education Department (GJJ13646), and the Postgraduate Innovation Fund of Jiangxi Province (YC2012-S121).

\section{References}

[1] P. Christou and R. M. Twyman, "The potential of genetically enhanced plants to address food insecurity," Nutrition Research Reviews, vol. 17, no. 1, pp. 23-42, 2004.

[2] FAO, The State of Food Insecurity in the World (SOFI), FAO, Rome, Italy, 2000, http://www.fao.org/FOCUS/E/SOFI00/ sofi001-e.htm.

[3] C. James, "Global food security," in International Congress of Plant Pathology, Pittsburgh, Pa, USA, August 1998.

[4] J. M. Bové, "Huanglongbing: a destructive, newly-emerging, century-old disease of citrus," Journal of Plant Pathology, vol. 88, no. 1, pp. 7-37, 2006.

[5] S. E. Halbert and K. L. Manjunath, "Asian citrus psyllids (Sternorrhyncha: Psyllidae) and greening disease of citrus: a literature review and assessment of risk in Florida," Florida Entomologist, vol. 87, no. 3, pp. 330-353, 2004. 
[6] S. Parnell, T. R. Gottwald, C. A. Gilligan, N. J. Cunniffe, and F. Van Den Bosch, "The effect of landscape pattern on the optimal eradication zone of an invading epidemic," Phytopathology, vol. 100, no. 7, pp. 638-644, 2010.

[7] R. F. Mizell III, C. Tipping, P. C. Andersen, B. V. Brodbeck, W. B. Hunter, and T. Northfield, "Behavioral model for Homalodisca vitripennis (Hemiptera: Cicadellidae): optimization of host plant utilization and management implications," Environmental Entomology, vol. 37, no. 5, pp. 1049-1062, 2008.

[8] G. A. Braga, S. Ternes et al., "Modelagem Matemática da Dinâmica Temporaldo HLB em Citros," in Proceedings of the 8th Congresso Brasileiro de Agroinformática, Bento Goncalves, 2011.

[9] D. G. Hall and M. G. Hentz, "Seasonal flight activity by the Asian citrus psyllid in east central Florida," Entomologia et Applicata, vol. 139, no. 1, pp. 75-85, 2011.

[10] X. Song and A. U. Neumann, "Global stability and periodic solution of the viral dynamics," Journal of Mathematical Analysis and Applications, vol. 329, no. 1, pp. 281-297, 2007.

[11] K. Wang, Z. Teng, and H. Jiang, "On the permanence for n-species non-autonomous Lotka-Volterra competitive system with infinite delays and feedback controls," International Journal of Biomathematics, vol. 1, no. 1, pp. 29-43, 2008.

[12] F. Zhang and X.-Q. Zhao, "A periodic epidemic model in a patchy environment," Journal of Mathematical Analysis and Applications, vol. 325, no. 1, pp. 496-516, 2007.

[13] T. Zhang and Z. Teng, "On a nonautonomous SEIRS model in epidemiology," Bulletin of Mathematical Biology, vol. 69, no. 8, pp. 2537-2559, 2007.

[14] Z. Teng and Z. Li, "Permanence and asymptotic behavior of the tv-species nonautonomous lotka-volterra competitive systems," Computers and Mathematics with Applications, vol. 39, no. 7-8, pp. 107-116, 2000.

[15] W. Wang and X.-Q. Zhao, "Threshold dynamics for compartmental epidemic models in periodic environments," Journal of Dynamics and Differential Equations, vol. 20, no. 3, pp. 699-717, 2008.

[16] Y. Nakata and T. Kuniya, "Global dynamics of a class of SEIRS epidemic models in a periodic environment," Journal of Mathematical Analysis and Applications, vol. 363, no. 1, pp. 230237, 2010.

[17] Y. Nakata, Permanence and Global Asymptotic Stability For Population Models in Mathematical Biology, Waseda University, Tokyo, Japan, 2010.

[18] H. Smith and P. Waltman, The Theory of the Chemostat, Cambridge University Press, Cambridge, Mass, USA, 1995.

[19] X. Zhao, Dynamical Systems in Population Biology, Spring, New York, NY, USA, 2003.

[20] X. M. Deng, "Formming process and basis and technological points of the theory emphasis on control citrus psylla for integrated control Huanglongbing," Chinese Agricultural Science Bulletin, vol. 25, no. 23, pp. 358-363, 2009 (Chinese).

[21] T. Li, C. Z. Cheng et al., "Detection of the bearing rate of liberobacter asiaticum in citrus psylla and its host plant," Acta Agriculturae Universitatis Jiangxiensis, vol. 29, no. 5, pp. 743745, 2007 (Chinese).

[22] G. F. Chen and X. M. Deng, "Dynamic observation adult citrus psyllid quantity live through the winter in spring and winter," South China Fruits, vol. 39, no. 4, pp. 36-38, 2010 (Chinese).

[23] X. M. Deng, G. F. Chen et al., "The newly process of Huanglongbing in citrus," Guangxi Horticulture, vol. 17, no. 3, pp. 49-51, 2006.
[24] R. G. d'A Vilamiu, S. Ternes, B. A. Guilherme et al., "A model for Huanglongbing spread between citrus plants including delay times and human intervention," in Proceedings of the International Conference of Numerical Analysis and Applied Mathematics (ICNAAM '12), vol. 1479, pp. 2315-2319, 2012. 


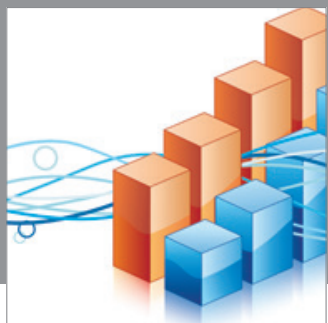

Advances in

Operations Research

mansans

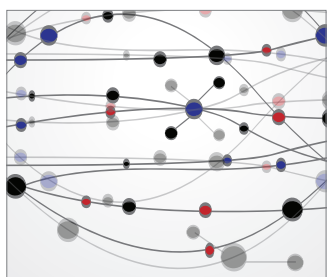

The Scientific World Journal
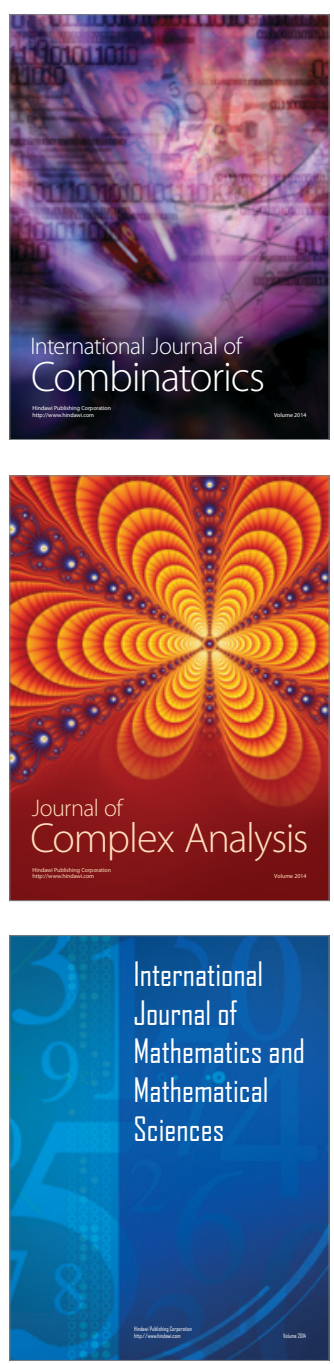
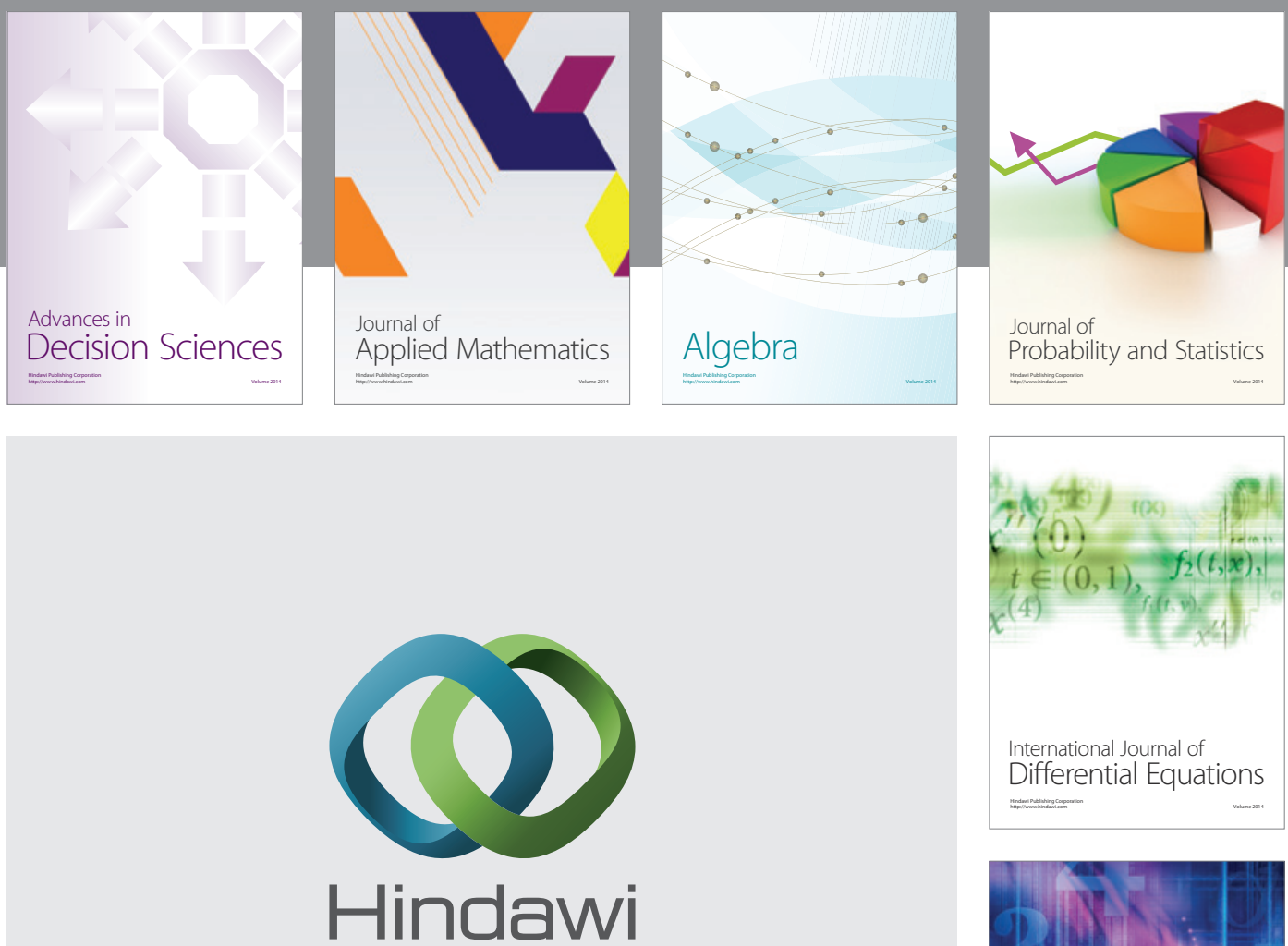

Submit your manuscripts at http://www.hindawi.com
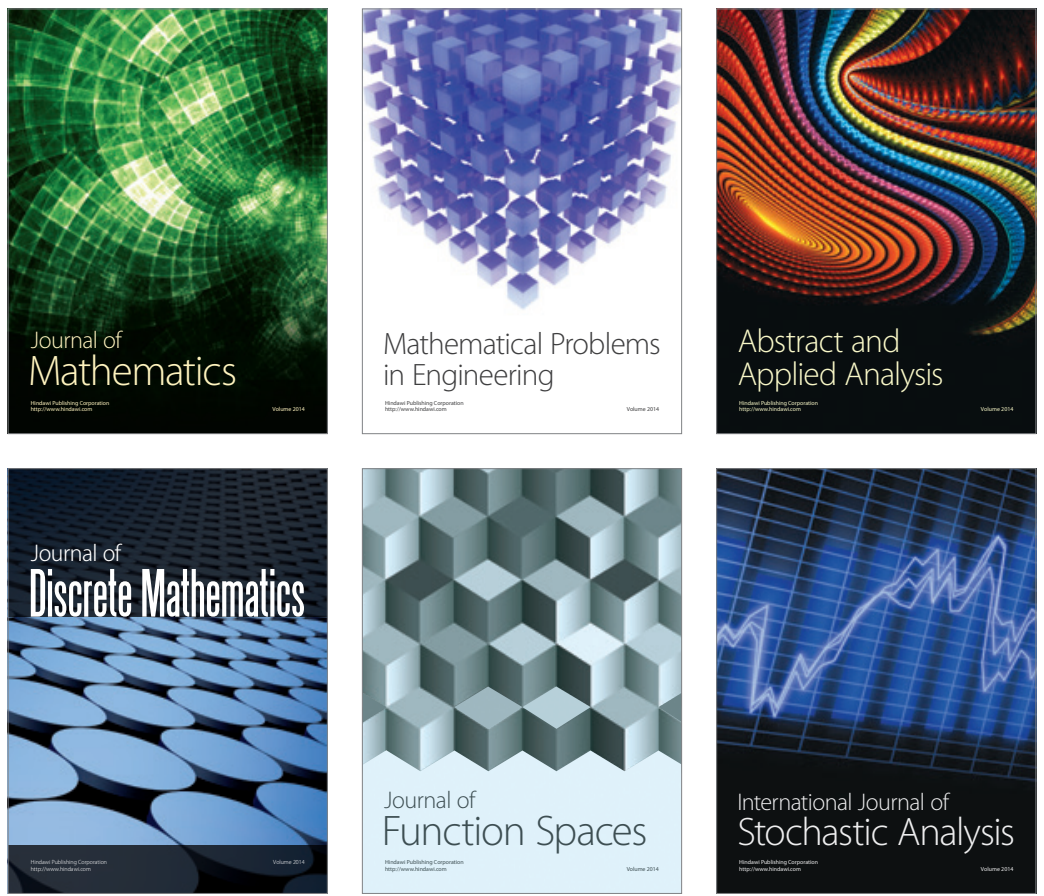

Journal of

Function Spaces

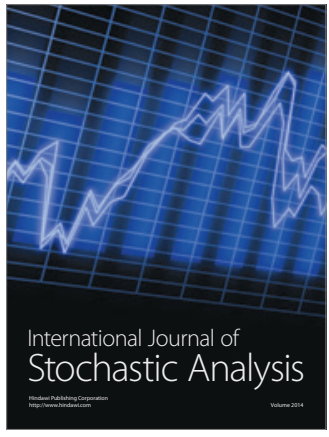

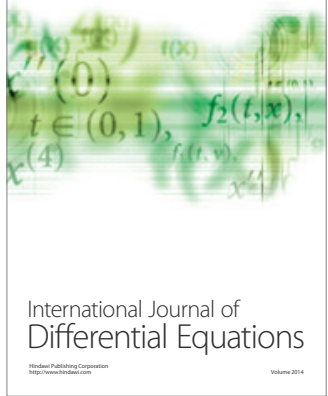
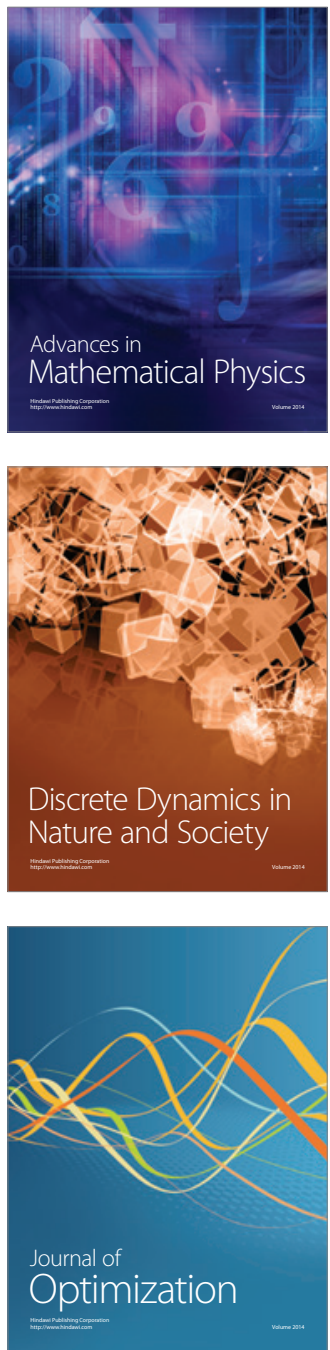